\title{
President's Statement
}

\author{
Hyeoun-Ae Park, President of IMIA
}

\section{Introduction}

It is a great honor for me to write the president's statement for 2017 IMIA Year Book on 'Learning from experience: Secondary use of patient data' as a special topic.

Healthcare providers around the world record millions of patients' health information in electronic health records (EHRs) every day. Researchers are using data from EHRs to answer their research questions on health services and public health. As the researchers extract knowledge from data collected during routine patient care and turn that knowledge into guidance for healthcare providers to use at the point of care, they help create what Lowrance called "learning from experience", [1] and the Institute of Medicine called "learning health care systems"[2]. Learning from experience in healthcare is a process with a series of continuous and iterative cycles - patient data is collected from clinical encounters, data is aggregated and analyzed, outcomes are evaluated, evidence is used to inform changes in care delivery, and practice is adjusted as needed for continual improvement $[2,3]$.

Since learning from experience studies real world patient care experiences, it can provide feedback to improve real world patient care experiences. It can enrich the findings of randomized control trials by allowing the study of subgroups of patients that can't be studied in a controlled trial. It can also help to find patterns of diagnostic accuracy, and assess conformity of practice with guidance by allowing working back from outcomes. Government agencies, health care organizations, and private companies are using data from EHRs and administrative claims to predict the risk of certain disease or adverse events, to conduct comparative effectiveness research, and to develop clinical practice guidelines and decision support tools for practicing clinicians. Research funding agencies are beginning to fund researches, which mine the data drawn from EHRs to advance medical knowledge. With the widespread adoption of health IT, the digital capture of health data, and big data analytics, the opportunities for learning from experience will only increase.

To foster learning from experience, a paradigm shift in healthcare is needed with continuous feedback loops between research and practice, and evidence and learning flowing in both directions. Whether these kinds of research involve a clinician using electronic databases to find an answer to his research problem or a data scientist using automated rules to identify patterns and trends in clinical big data, it requires informatics and data science for real time capture of the patient care experience, generation of knowledge, and access to knowledge.

\section{Technical and Ethical Challenges}

Learning from experience in healthcare is very different from that of other fields because healthcare data lacks uniformity and is highly sensitive. There are some technical and ethical challenges to achieving successful learning from experience due to these unique characteristics of healthcare data. Each of these challenges is explored below.

The use of EHRs is a key element of learning from experience. However, the lack of uniformity among healthcare data is one barrier [4]. Healthcare data tends to reside in multiple systems like electronic medical records (EMRs) or picture archiving and communication systems (PACSs), and in multiple places like radiology or pharmacy. Healthcare data exists in different formats (e.g., text, numeric, images, videos, multimedia, etc.). Sometimes the same data exists in different systems and in different formats. For example, a patient's broken leg appears as an image in the medical record, but appears as an ICD-9 code in claims data. Healthcare data has inconsistent or variable definitions. For example, physicians define a cohort of depressive patients differently than nurses. Multiple clinicians from different groups may have inconsistent 
views of the treatment for a same condition. Healthcare data is an amalgam of complex individual systems. Claims data has been around for years and it has been standardized, however it is incomplete. Clinical data from EMRs gives a more complete picture of the patient's experience, however it is not always standardized.

The secondary use of EHR data has the potential to transform health care. However, in order to realize the transformative potential of EHRs, healthcare organizations need to be able to exchange and share data each other in a way that is timely and secure. EHRs data also needs to be interoperable with other healthcare databases (e.g., cancer registries). However, health care organizations have routinely adopted health IT systems customized to local institutional needs, which makes communication with other organizations difficult.

Another challenge is the protection of personal data in the case of the secondary use of data in health research. In most countries, patients' personal data, including patients' health and genetic data, cannot be shared unless the patient gives explicit and unambiguous consent to the use of his/her data or there is a public interest mandate. Although informed consent is an essential process, exemptions to consent for sharing data are needed to make research possible in some cases. In this case, researchers must ensure that technical and organizational safeguards are in place when using patients' data [5].

One such safeguard is anonymization which removes the association between the identifying data set and the data subject. This is the most common and widely accepted method of data protection in health research and secondary use of data. Another safeguard is pseudonymization which replaces the identifying data with one or more pseudonyms. This will often be achieved by attaching codes to the identifying data so that the data will only be identifiable to those who have access to the key. Another safeguard is to maintain the confidentiality of patient information. Confidentiality involves a set of rules that limit access to or place restrictions on certain types of information. The ethical principle of confidentiality requires that personal information shared by a patient with a healthcare provider in the course of patient care is not shared with others.

\section{Strategies to Foster Learning from Experience}

Despite these challenges, learning from experience has the potential to transform health care. Strategies to foster learning from experience include enabling interoperability among institutions and individuals that support care delivery across the care continuum, facilitating data sharing and integration to improve care and advance research, and enabling patient and public engagement.

Learning from experience relies on the efficient and effective flow of data among all stakeholders. The potential benefits of interoperable systems are particularly great for learning from experience because health research depends on the access to accurate and complete data. To accomplish this, information technology (IT) industry and government agencies are working together to create the interoperability ecosystem to promote not only technical interoperability but also organization and semantic interoperability [6].

Vast amounts of healthcare data are being accumulated. If data from various sources were integrated and made broadly available to researchers, far greater insights would be possible [7]. Multiple initiatives are under way to facilitate data sharing among clinicians and researchers (e.g. Genomic Data Commons and Oncology Research Information Exchange Network [ORIEN]). Rich data sets could also be created by linking large databases housing different types of data, such as clinical data from EHRs, population-based health survey results, surveillance data, behavioral data, environmental data, and claims data. Integration of these complementary data can facilitate more meaningful and comprehensive analyses of outcomes, patterns of care, cost-effectiveness, social determinants of health, health services, and other factors.

The patient is an active contributor to the learning from experience process $[2,8]$. The active engagement of the patient is viewed as a patient-centered activity. The Institute of Medicine (IOM) describes this as "patient-anchored care". In the patient-anchored care environment, each patient-clinician interaction starts with the uninterrupted attention to the patient's voice on issues, perspectives, goals, and preferences. With best practice as the starting point, evidence-based tailoring is informed by patient's biological traits, circumstances, and preferences. All relevant aspects of the patient care experience, including patient perspectives, should be captured and routinely assessed against expectations. Patient engagement will be more critical to the success of the learning process as the role of genetic and "omics" technologies and personalized medicine increases.

Given the opportunities, challenges, and strategic items outlined above, this is the right time for IMIA to have 'Learning from experience: Secondary use of patient data' as the topic of the 2017 IMIA Year book. I hope IMIA as a leading international organization on health and biomedical informatics uses this opportunity to spearhead the movement of learning from experience globally. IMIA has a group of experts actively participating to the various Working Groups and Special Interest Groups (WG/SIGs) which can address different aspects of learning from experience.

\section{References}

1. Lowrance WW. Learning from experience: privacy and the secondary use of data in health research. J Biolaw Bus 2003;6(4):30-60.

2. Institute of Medicine. Best care at lower cost: the path to continuously learning health care in America. Smith M, Saunders R, Stuckhardt L, McGinnis JM, editors. Washington (DC): The National Academies Press; 2013. Available from: http://nap.edu/13444

3. Greene SM, Reid RJ, Larson EB. Implementing the learning health system: from concept to action. Ann Intern Med 2012; 157(3):207-10.

4. Raghupathi W, Raghupathi V. Big data analytics in healthcare: promise and potential. Health Inf Sci Syst 2014;2:3.

5. Rentzepopoulos P. Secondary Use of Health Data for Medical Research and Public Health. eHealth Procurers Forum. https://joinup.ec.europa.eu/ community/ehealthprocurers/document/secondaryuse-health-data-medical-research-and-public-health

6. Office of the National Coordinator for Health Information Technology. Connecting health and care for the nation: a shared nationwide interoperability roadmap. Washington (DC): ONC; 2015 Oct. Available at: https://www.healthit.gov/sites/ default/files/hie-interoperability/nationwide-interoperability-roadmap-final-version-1.0.pdf

7. Foley T, Fairmicheal F. The potential of learning healthcare systems. The Learning Healthcare Project. 2015. Available from http://www.learninghealthcareproject.org/LHS_Report_2015.pdf

8. Seid M, Margolis PA, Opipari-Arrigan L. Engagement, Peer Production, and the Learning Healthcare System. JAMA Pediatr 2014;168(3):201-2.

http://dx.doi.org/10.15265/IY-2016-028 